\title{
Endogenous visuospatial precuing effects as a function of age and task demands
}

\author{
DONALD J. TELLINGHUISEN \\ Augustana College, Sioux Falls, South Dakota \\ and \\ LYNN D. ZIMBA and DONALD A. ROBIN \\ University of Iowa, Iowa City, Iowa
}

\begin{abstract}
This experiment examined the effects of age on processing resource capacity using an endogenous visuospatial precuing task and four levels of resource demands. Younger and older adults made speeded two-choice responses to dim and bright targets that required a line-orientation or a lexical decision. An arrow preceding target onset served as an attentional cue to affect the spatial distribution of resources. It provided accurate information about the target's location on most trials and inaccurate or neutral information on the remaining trials. Although older adults were slower than younger adults under all conditions and were more affected by the resource demand manipulations, they exhibited a pattern of precuing effects across conditions that was similar to that of the younger adults. Results are consistent with the idea that the visuospatial attention system remains relatively unaffected by aging. However, the data speak against the idea that capacity reduction is the primary contributor to age-related slowing.
\end{abstract}

One of the most pervasive findings in cognitive aging research is that there is an increased slowness of behavior with increased age. As Wickens, Braune, and Stokes (1987) noted, the evidence appears unambiguous that there is a slowing in speed of performance for individuals above the age of 25 on information-processing tasks in which response latency (RT) is the dependent measure. This slowing has been attributed to age effects at nearly all stages of information processing (see Kausler, 1991 , for a review), although certain processing stages may be affected to a greater or lesser extent than others (e.g., Allen, Madden, Weber, \& Groth, 1993; Lima, Hale, \& Myerson, 1991; Maylor \& Rabbitt, 1994). The magnitude of the age difference in performance speed has typically been found to increase with increases in the process-

The research was supported by a University of lowa Aging Seed Grant and NIH BRS Grant 2S07RR0735-23 awarded to L.D.Z., a Sigma Xi Grant-in-Aid of Research awarded to D.J.T., and NINDS Grant PO-NS19632, on which D.A.R. is an investigator. This article is based on a master thesis by D.J.T. under the supervision of L.D.Z. The research was conducted in the Department of Psychology at the University of Iowa. Filot data for this study were collected by Melissa Brewer in partial fulfillment of her honor's research thesis. Portions of this research were presented at the 32nd Annual Meeting of the Psychonomic Society, November 1991, in San Francisco. The technical assistance of Kevin Schartz, Keith Miller, and Lloyd Frei is gratefully acknowledged, as is the statistical advice of Don Dorfman and George Woodworth. The authors wish to acknowledge the helpful critiques of Art Kramer, Alan Hartley, Darryl Humphrey, and an anonymous reviewer on previous versions of this manuscript. Correspondence should be addressed to L. D. Zimba, Laboratory of Speech and Language Neuroscience, Department of Speech Pathology and Audiology, University of Iowa, 13 WJSHC, Iowa City, IA 52242-1407 (e-mail: lynn-zimba@uiowa.edu). ing demands of the task. Salthouse $(1988,1991,1992 a)$ suggested that the slowing phenomenon may result from any one or a combination of three process-oriented factors: age-related differences in the efficiency of one or more task-specific information-processing components, strategic differences in the usage of components, and a reduction in the quantity of processing resources available for task performance. Furthermore, he noted that, on the basis of the available data, resource reduction may be the primary contributor to the slowing phenomenon.

Processing resources are considered to be facilities essential for information processing. Initially, they were conceptualized as an undifferentiated capacity-limited commodity (resource pool) that could be distributed in graded quantities among different processing stages (e.g., Kahneman, 1973; Moray, 1967; Shaw, 1978; Shaw $\&$ Shaw, 1977). More recent reports, however, postulate multiple capacity-limited pools of processing resources rather than a unitary resource pool (e.g., Navon \& Gopher, 1979, 1980; Wickens, 1980, 1984, 1987). For example, Wickens $(1980,1984)$ presented a multiple-resource model in which separate resource pools are defined by three dichotomous dimensions consisting of processing stages (perceptual-central vs. response), input modalities (visual vs. auditory), and processing codes (verbal/ linguistic vs. spatial/analog). Resources can be distributed to the extent that the processes involved utilize resources from the same resource pool. Navon and Gopher $(1979,1980)$ provided a conceptual framework for examining the relationship between processing resources and RT performance. In general, they proposed that the latency to achieve a criterion level of performance is a function of the amount of processing resources invested 
per unit time from each of the relevant resource pools, the average contribution (processing efficiency) of a unit of invested resources, and specific subject-task parameters, such as stimulus quality, predictability of the stimulus event, response complexity, level of practice, and manual dexterity, that impose constraints on the system. Resources are distributed by the system according to an allocation policy determined by the task demands and the situational priorities, but within the limits of the availability of resources per unit time (the capacity) of each of the relevant resource pools.

A reduction in the capacity of one or more resource pools would be expected to impact the ability of the system to meet the intended levels of performance. A performance decrement would be incurred, and this decrement should be evident on tasks in which resources are allocated to multiple sources of information. However, the study of resource allocation as a function of aging has not always produced results consistent with this prediction. For example, consistent age-related differences in resource allocation have not been observed in studies involving visuospatial precuing (e.g., Folk \& Hoyer, 1992; Hartley, Kieley, \& Slabach, 1990; Nissen \& Corkin, 1985; Posner, Inhoff, Friedrich, \& Cohen, 1987; Robin \& Rizzo, 1992; Robinson \& Kertzman, 1990). These studies employed techniques similar to that used by Posner and his colleagues (e.g., Posner, Snyder, \& Davidson, 1980). On a majority of trials, an informative visual precue specifies a location in the visual field, other than the point of fixation, that is most likely to contain an upcoming target. Most of these trials are valid because the target appears at the specified location; the remainder of these trials are invalid because the target appears at a location other than the one specified. On a minority of trials, a noninformative visual precue is presented that does not provide specific information about the target's location. These trials are neutral in that the target has an equal likelihood of appearing at any one of a number of predetermined locations. The precue can represent the cued location symbolically (an endogenous precue), or it can occur at or near the cued location (an exogenous precue). The dependent variable is the RT to detect or identify the target. It is usually found that validtrial RTs are fastest, neutral-trial RTs are slower, and invalid-trial RTs are slowest. These performance differences are typically attributed to a differential spatial distribution of processing resources initiated in response to the precue and controlled by a subsystem of attention (e.g., Henderson, 1991; Johnson \& Yantis, 1995; LaBerge \& Brown, 1989). ${ }^{1}$ The sensitivity of this attentional subsystem to various task parameters, such as target salience (e.g., Hawkins, Shafto, \& Richardson, 1988) and feature arrangement (e.g., Cheal \& Lyon, 1992), predictive validity of the precue (e.g., Hughes, 1984), and spatial extent of the precued area (e.g., Eriksen \& Yeh, 1985; Henderson, 1991), is reflected in different performance profiles across valid, neutral, and invalid trials.
If aging incurs a reduction in processing resource capacity, then the performance profile would be expected to differ between groups of younger and older individuals, particularly under conditions in which performance criteria (e.g., a rapid rate of responding with a high level of accuracy) are maintained. For example, to meet performance criteria on valid trials, older individuals might strategically allocate a greater share of resource capacity to the processing of targets originating at the most probable location and, thus, a lesser share of resource capacity to the processing of targets originating at less probable locations. This would result in a larger RT difference between valid and invalid trials for older individuals than for younger individuals. On the other hand, older individuals might adopt the same allocation policy as younger adults. In this case, the reduced capacity of older individuals would be expected to yield a smaller difference between valid and invalid trial RTs and, possibly, a higher error rate. Other patterns of differences could be hypothesized as well. But clear age differences in the pattern of performance on valid, invalid, and neutral trials have been consistently absent, and this has raised questions about the generality of resource reduction as an explanation of age-related cognitive slowing (e.g., Folk \& Hoyer, 1992; Hartley, 1993; Nissen \& Corkin, 1985).

There is one possibility, however, that could account for the lack of obtained age differences in precuing effects. Navon and Gopher $(1979,1980)$ and others (e.g., Kahneman, 1973; Wickens, 1984) suggested that the amount of resources invested in task performance depends on the demand for those resources. Resource demand is dependent on the task performance criteria and the specific subject-task parameters. It may be that the stimuli and/or the tasks utilized in previous studies did not require resources to the extent necessary to reveal age-related differences in capacity. All of the studies except those of Robin and Rizzo (1992) and Posner et al. (1987) employed very salient, relatively simple targets and only a low level of task complexity, where complexity refers to the number of processing steps required to complete the task. Also, all of the studies except those of Hartley et al. (1990) and Folk and Hoyer (1992) required simple detection responses (SRTs). Hartley et al. required observers to respond to one of two possible targets and to withhold responses to the other, whereas Folk and Hoyer examined the ability to differentiate " =" from " $\times$." Madden (1992) used a task involving both spatial precuing and visual search (locating and identifying a target letter among nontarget letters). He obtained age differences in precuing effects, but the differences were due to a difference in the time course of cue utilization rather than to a difference in capacity. Robin and Rizzo (1992) manipulated task complexity by presenting targets in either a unimodal condition in which target modality (auditory or visual) was constant or a mixedmodal condition in which target modality was uncertain. SRTs were longer in the mixed-modal condition than in 
the unimodal condition, but the increase in task complexity did not result in age differences in precuing effects. Posner et al. (1987) compared precuing effects in a SRT task performed under single- and dual-task conditions. Although age was a factor in the experiment, agerelated differences in precuing effects were not reported.

In these studies, the resource demand and, thus, the resource investment, may have been less than the resource capacity of the participants. This would have obscured differences in capacity and, likewise, differences in precuing effects. The aim of the present study was to test the hypothesis that, because of age-related resource reduction, age differences would be observed in the pattern of performance on valid, invalid, and neutral trials in a visuospatial precuing task. To examine the possibility that low levels of resource demands may have obscured age differences in previous studies, valid-, neutral-, and invalid-trial RTs were measured under conditions presumed to vary in resource requirements. This methodof measuring performance under conditions of varied resource demands-was recommended by Salthouse (1988) for examining age-related capacity reduction.

Resource demands varied across four conditions that included two levels of task complexity--a line-orientation decision task and a lexical decision task-and, within each level of task complexity, two levels of target luminance - dim and bright targets. The line-orientation decision task, in which subjects signaled whether a line segment was vertically or horizontally oriented, represented a lower level of complexity, comparable to the less-demanding tasks of previous studies. A correct response involved a discrimination based on only the physical characteristics of the stimulus. In the lexical decision task, subjects signaled whether a letter string composed a word or a pseudoword (a letter string that is pronounceable but does not have an English meaning). This task represented a higher level of complexity, because a correct response could not be made on the basis of the physical characteristics of the stimulus alone but required a semantic decision as well. It was expected that the additional processing requirements of the lexical task would increase the demand for resources relative to the line-orientation task. However, it was unclear whether resource demands would be sufficiently increased by this manipulation to reveal age differences in resource capacity. Although the results of several studies indicate that older adults take significantly longer to recognize visually presented words than do younger adults (e.g., Allen, Madden, \& Crozier, 1991; Bowles \& Poon, 1981), Lima et al. (1991) concluded that performance on lexical tasks (i.e., tasks utilizing words as stimuli) is less affected by age than is performance on nonlexical tasks.

Allen et al. (1993) reported that age differences in word recognition latencies may be due more to age decrements in peripheral processing stages rather than central processing stages. Using a lexical decision task, they found significant age differences for manipulations of stimulus encoding and response selection load but not for manipulations of word frequency, an index of lexical access. Because, in the present study, the most salient difference between the line-orientation and the lexical tasks was with regard to the amount of central processing requirements, a luminance manipulation was included in order to vary peripheral processing requirements as well. Compared with younger adults, older adults view stimuli at an overall reduced luminance due primarily to the deterioration of optical components in the eye (e.g., Kline \& Schieber, 1985). Weale $(1963,1982)$ estimated that this reduction could be as great as two thirds (i.e., older adults may experience as little as one third of the average luminance experienced by younger adults). Thus, it was expected that variations in target luminance would have a greater impact on resource demands for older adults than for younger adults.

Finally, it was assumed that variations in resource demands produced by the task complexity and target luminance manipulations would affect the same limitedcapacity resource pools, which, according to Wickens's $(1980,1984)$ multiple-resource model, are defined by perceptual-central processing stages, visual input modality, and verbal/linguistic processing codes. This was an important consideration for satisfying the objectives of the study. In general, it was expected that, while age differences in visuospatial precuing effects might be absent under conditions of low resource demands comparable to those of previous studies (e.g., bright targets in the line-orientation task), differences would emerge under conditions of increased resource demands (e.g., dim targets in the lexical task). No specific predictions were made regarding the point at which these differences would emerge.

\section{METHOD}

\section{Participants}

Fifteen students ( 8 males, 7 females) attending the University of Iowa between the ages of 20 and 27 years (mean age $=22.4$ years) volunteered to participate in the study. These individuals composed the younger group. Fifteen adults ( 9 males, 6 females) between the ages of 64 and 68 years (mean age $=65.1$ years) were recruited from the Iowa City community. These individuals composed the older group. Four persons in the older group had parttime jobs, 1 had a full-time job, and the other 10 were active in volunteer organizations; all described themselves as active. Individuals in the older group had an average of 16.2 years of formal education (all had earned at least a high school diploma); those in the younger group had an average of 16.1 years of formal education. All of the participants were right-handed. Individuals in the older group received payment for their participation in the study.

The participants were screened for major health and visual problems, including double vision, glaucoma, cataracts, dry eyes, amblyopia, achromatism, diabetes, and general visual dysfunction, and were required to have had their last refraction within a year. In addition, all participants had to attain an accuracy rate of $95 \%$ on practice tasks (see Procedure section). It was reasoned that if individuals could achieve this level of performance, they were able to adequately view the target stimuli, were able to perform the task at hand, and, thus, were fit participants for the experiment. Two potential participants in the older group were eliminated because of poor vision. Two participants withdrew from the 
experiment for personal reasons; they were replaced by 2 other participants. Six of the 15 individuals in the younger group participated in a similar pilot experiment approximately 3 months before this experiment. All of the individuals in both groups participated in another pilot experiment approximately $1-2$ weeks prior to participating in this experiment. The pilot experiment was identical to this experiment except that the visual precue always provided accurate information about the location of the upcoming target. Individuals completed the present experiment over the course of three or four 2-h sessions; no individual participated in more than one session per day.

\section{Stimulus Display and Apparatus}

A Mitsubishi 13-in. video monitor controlled by a Mitsubishi MP 386 microcomputer was used to generate the display. The monitor was $57 \mathrm{~cm}$ from a chin-and-head rest used to stabilize head position. Testing was conducted in a dark room. The display consisted of a central fixation cross $\left(0.8^{\circ} \times 0.9^{\circ}\right)$, an (endogenous) arrow precue $\left(0.8^{\circ} \times 0.6^{\circ}\right)$ located $1.4^{\circ}$ from the fixation cross, and a visual target. Targets were either line segments or letter strings, and these were centered $3^{\circ}$ above or below the center of the fixation cross. Line segments were either vertical or horizontal, $0.8^{\circ}$ long and $0.1^{\circ}$ wide. Letter strings were composed of three, four, or five letters. The letters, generated from the Helvetica font of Microsoft Quick Pascal, varied in width from $0.2^{\circ}$ to $0.8^{\circ}$ (depending on the letter) and were $0.8^{\circ}$ high. Lines composing the letter strings were $0.2^{\circ}$ wide. Letter strings ranged from $2.5^{\circ}$ to $5.5^{\circ}$ in length. These large stimulus sizes were used to minimize any potential group differences in acuity.

Letter strings formed either words or pseudowords. Words were selected from The American Heritage Word Frequency Book (Carroll, Davies, \& Richman, 1971), which contains 86,741 different words drawn from 1,045 published texts. Only words with a standard frequency index (SFI) of above 50 were used. The SFI relates to the frequency per million of a word. Pseudowords were generated from the words by changing one or two letters. One list of 25 words and 25 pseudowords was used for practice trials. Twelve lists of 120-125 letter strings were used for test trials; six of these contained words and 6 contained pseudowords.

Two levels of target luminance were generated by utilizing two predefined brightness codes in Quick Pascal: high-luminance targets consisted of character No. 7 (light gray), and low-luminance targets consisted of No. 8 (dark gray). A high-luminance line stimulus was $31 \mathrm{mfL}$; a low-luminance line stimulus was $1 \mathrm{mfL}$. The luminance of a single letter (the letter $I$ ) was $62 \mathrm{mfL}$ in the high-luminance condition and $2 \mathrm{mfL}$ in the low-luminance condition. The fixation cross and precues were red (No. 4). The luminance of a single arrow precue was $12 \mathrm{mfL}$; the luminance of the fixation cross was $53 \mathrm{mfL}$. All stimuli were presented against a black background.

A response box with three keys (microswitches) was situated in front of the participant. Responses were made by pressing either the left key or the right key, and these were polled for response every millisecond using a clock-driven interrupt routine. The third key, located in the center, was used to initiate a trial block. To ensure fixation, eye movements were monitored using Ag$\mathrm{AgCl}$, SynCor Neonatal recording electrodes that were dc coupled. The signals from the electrodes were digitized (sampling rate of $40 \mathrm{~Hz}$ ) after amplification. The EOG had a sensitivity of $1.5^{\circ}$; deviations from fixation that exceeded $1.5^{\circ}$ before target onset automatically reset the trial. Trials on which an eye movement occurred during target presentation were rerun at the end of the block. Auditory feedback signaled that an eye movement was detected.

\section{Procedure}

Each individual was tested under three experimental manipulations: two levels of task complexity (line-orientation decisions, lexical decisions), two levels of target luminance (dim, bright) for each task, and three types of trial (valid, invalid, neutral). The level of task complexity was held constant throughout a block of trials; the luminance levels and trial types were mixed within trial blocks. There was a total of 10 test blocks and a minimum of 2 practice blocks. Every block began with an EOG calibration procedure. Each individual then pressed the center response key to initiate a series of trials. The fixation mark appeared and remained present throughout the block. The intertrial interval was $3 \mathrm{sec}$, followed by a $200-\mathrm{msec}$ ready tone and a $200-\mathrm{msec}$ delay, and then a precue was presented for $200 \mathrm{msec}$. The interval between the onset of the precue and the target onset (SOA) varied randomly between 1,000 and $2,000 \mathrm{msec}$. The target remained on the screen until the observer made a response. This procedure avoided the possibility of age differences in performance due only to exposure duration (Somberg \& Salthouse, 1982). A 2-min rest interval was provided midway through the block.

Within each block, there were three types of trial: valid, invalid, and neutral. On valid trials, a single arrow precue was presented, and the target subsequently appeared at the location indicated by the arrow. Thus, if the arrow pointed up, the target appeared above the fixation mark, and if the arrow pointed down, the target appeared below the fixation mark. On invalid trials, a single arrow precue was also presented, but the target appeared at the location opposite to that indicated by the arrow. On neutral trials, two arrows were presented - one pointing up and one pointing downand the target appeared above or below fixation with equal frequency. Of the 10 test blocks, 4 were devoted to testing on the line-orientation task and 6 were devoted to testing on the lexical task. Each test block of the line-orientation task consisted of 192 trials. On half of the trials, the target type was horizontal; on the other half of the trials, it was vertical. Each block of the lexical task consisted of 240 trials. On half of the trials, the target type was a word; on the other half of the trials, it was a pseudoword. ${ }^{2}$ Of the total trials in a block, $16.7 \%$ were neutral; of the remaining trials, $80 \%$ were valid and $20 \%$ were invalid. Target type, target location, and target luminance were distributed evenly over the three types of trial. The order of the 10 test blocks was randomized for each participant, and the order of trials was randomized for each block.

The response task was to correctly identify the target as either vertically or horizontally oriented if it was a line segment or as a word or a pseudoword if it was a letter string. The participants were instructed to respond as quickly and accurately as possible, and both choice RT (CRT) and accuracy were recorded. Responses were made by pressing one of the two designated response keys. Half of the individuals in each group were assigned one of two response key designations: (1) left key if a horizontal line or a pseudoword appeared and right key if a vertical line or word appeared, or (2) left key if a vertical line or word appeared and right key if a horizontal line or pseudoword appeared. The individuals responded with the left hand for left-key responses and with the right hand for right-key responses.

Practice blocks were administered prior to test blocks. Practice blocks were identical to test blocks except they consisted of only 96 trials. The practice blocks also served as a screen for eligibility in the experiment: The participants were required to correctly identify the target on at least $95 \%$ of the trials. Practice blocks were repeated until the participant achieved this criterion. No participant required more than three practice blocks of either task to reach criterion, and no participants were rejected due to inability to achieve the performance criterion. Words and pseudowords were drawn randomly with replacement from the practice lists on practice blocks. On the six test blocks, words were chosen randomly without replacement from one of the six word lists, and pseudowords were chosen randomly without replacement from one of the six pseudoword lists. For each observer, one word list 
and one pseudoword list were selected for each block of trials. The lists were not repeated, and the order of presentation was random with the restriction that, because the pseudowords were derived from the words in each list, words and pseudowords from corresponding lists were not presented in the same block.

\section{RESULTS}

Mean CRTs ${ }^{3}$ from trials with correct responses were calculated for each observer under each condition, and these were statistically analyzed using an analysis of variance (ANOVA). An arcsin transformation of the mean proportion of correct responses (Kirk, 1982) was performed for each observer under each condition, and these data were also analyzed by ANOVA. All tests of significance were conducted at the .05 level.

\section{Response Latency}

A four-factor ANOVA was conducted on the latency data. Age (younger, older) was a between-subjects factor, and trial type (valid, neutral, invalid), task complexity (line-orientation decision, lexical decision), and target luminance (bright, dim) were within-subjects factors. Because the average luminances of the target stimuli in the line-orientation task and those in the lexical task were different depending on the task (see Method section), the target luminance factor was nested within the task-complexity factor. This procedure eliminated evaluation of any interaction effects involving task complexity and target luminance. [The nesting of luminance within task is denoted as luminance(task).]

The means and standard errors for each of the experimental conditions are provided in Table 1 . The analysis revealed significant main effects of age $[F(1,28)=53.34]$, trial type $[F(2,56)=43.76]$, task complexity $[F(1,28)=$ 189.62 , and luminance (task) $[F(2,56)=42.04]$ (all $p$ s $<$ $.001)$. In addition, four of the five two-way interactions

Table 1

Mean Choice Response Time $(M)$ and Standard Error $(S E)$ (in Milliseconds) as a Function of Age, Trial Type, Task Complexity, and Target Luminance

\begin{tabular}{|c|c|c|c|c|c|c|}
\hline \multirow{3}{*}{$\begin{array}{c}\text { Target } \\
\text { Luminance }\end{array}$} & \multicolumn{6}{|c|}{ Trial Type } \\
\hline & \multicolumn{2}{|c|}{ Valid } & \multicolumn{2}{|c|}{ Neutral } & \multicolumn{2}{|c|}{ Invalid } \\
\hline & $M$ & $S E$ & $M$ & $S E$ & $M$ & $S E$ \\
\hline \multicolumn{7}{|c|}{ Line-Orientation Task } \\
\hline \multicolumn{7}{|c|}{ Younger adults } \\
\hline Bright & 458 & 20 & 475 & 20 & 500 & 17 \\
\hline Dim & 508 & 20 & 536 & 24 & 544 & 18 \\
\hline \multicolumn{7}{|l|}{ Older adults } \\
\hline Bright & 639 & 26 & 658 & 26 & 681 & 25 \\
\hline Dim & 715 & 29 & 764 & 38 & 747 & 27 \\
\hline \multicolumn{7}{|c|}{ Lexical Task } \\
\hline \multicolumn{7}{|l|}{ Younger adults } \\
\hline Bright & 625 & 17 & 652 & 15 & 693 & 16 \\
\hline Dim & 651 & 18 & $67 \overline{7}$ & 15 & 727 & 19 \\
\hline \multicolumn{7}{|l|}{ Older adults } \\
\hline Bright & 911 & 42 & 960 & 41 & 999 & 41 \\
\hline Dim & 1,033 & 61 & 1,085 & 63 & 1,124 & 53 \\
\hline
\end{tabular}

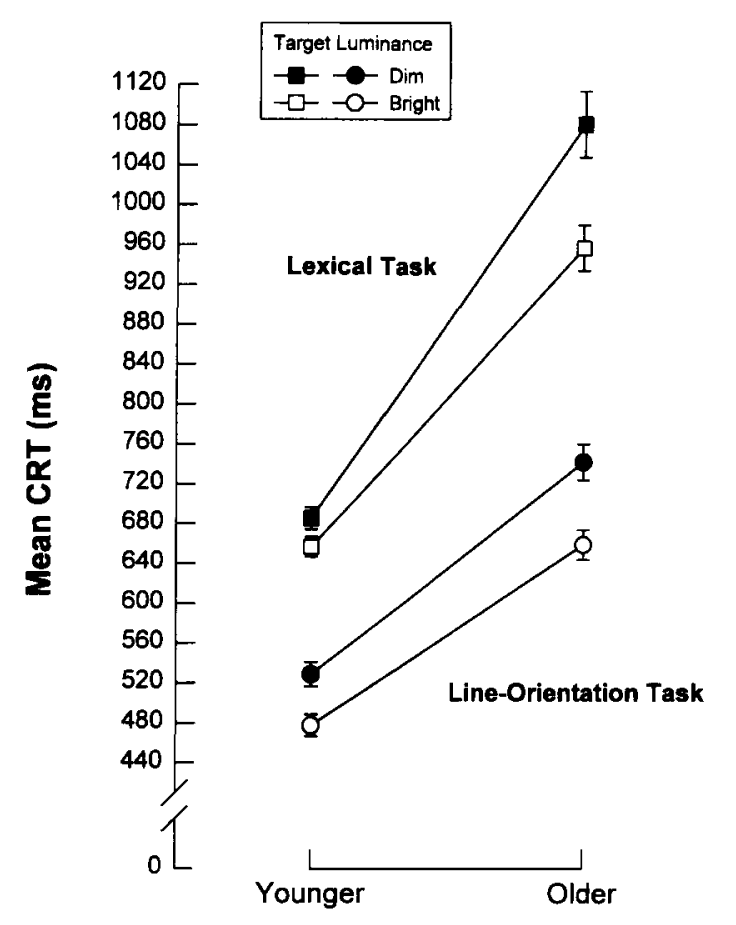

Age Group

Figure 1. Mean CRTs of the younger and older age groups in the lexical decision task (squares) and the line-orientation decision task (circles) with bright luminance targets (unfilled symbols) and dim luminance targets (filled symbols). Bars $= \pm 1$ SEM.

were significant [age $\times$ task complexity, $F(1,28)=18.33$, $p<.001 ;$ age $\times$ luminance(task), $F(2,56)=10.37, p<$ .001 ; trial type $\times$ luminance (task), $F(4,112)=3.05$, $p<.02$; trial type $\times$ task complexity, $F(2,56)=13.15$, $p<.001]$. None of the interactions involving age and trial type were significant [age $\times$ trial type, $F(2,56)=0.95$, $p>.39$; age $\times$ trial type $\times$ task complexity, $F(2,56)=0.66$, $p>.51$; age $\times$ trial type $\times$ luminance $($ task $), F(4,112)=$ $0.58, p>.67]$.

Means from the significant age $\times$ luminance(task) interaction are plotted in Figure 1. Separate follow-up ANOVAs were conducted on the data from each task. The analyses revealed a significant age $\times$ luminance interaction both for the line-orientation task (Figure 1, circles) $[F(1,28)=9.85, p<.01]$ and for the lexical task (Figure 1 , squares) $[F(1,28)=10.43, p<.01]$. Additional follow-up tests of these interactions revealed the same pattern of results for both tasks. The older adults' performance was significantly slower than that of the younger adults under each level of target luminance, and, although the luminance manipulation significantly affected the performance of both groups, the older adults were significantly more affected by this manipulation than were the younger adults. In the line-orientation task, the mean effects of luminance were $52 \mathrm{msec}$ for the younger adults and $83 \mathrm{msec}$ for the older adults; in the 
lexical task, the effects of luminance were $28 \mathrm{msec}$ and $124 \mathrm{msec}$ for the younger and older adults, respectively.

A similar set of results was obtained with respect to the significant age $\times$ task complexity interaction, which is also evident in Figure 1. Follow-up tests revealed that older adults responded significantly slower than did younger adults in both tasks, and, although the effect of task was significant for both younger and older adults, the older adults were significantly more affected by the task manipulation than were the younger adults. The mean effects of task were $167 \mathrm{msec}$ for the younger adults and $318 \mathrm{msec}$ for the older adults.

The significant trial type $\times$ luminance(task) interaction is depicted in Figure 2. Separate follow-up ANOVAs were conducted on the data from each task. For the lexical task (Figure 2, squares), there was a significant effect of luminance $[F(1,28)=26.29, p<.001]$ and trial type $[F(2,56)=39.59, p<.001]$, but the interaction was not significant $[F(2,56)=0.35]$. The effects of luminance in this task were 74,75 , and $80 \mathrm{msec}$ for valid, neutral, and invalid trials, respectively. For the line-orientation task (Figure 2, circles), there was a significant effect of luminance $[F(1,28)=182.14, p<.001]$ and trial type $[F(2,56)=21.49, p<.001]$, and there was a significant interaction $[F(2,56)=4.36, p<.02]$. Follow-up tests of this interaction indicated that the effect of trial type was significant for both levels of target luminance and that the effect of luminance was significant for each level of trial type. Bonferroni comparisons of the magnitude of

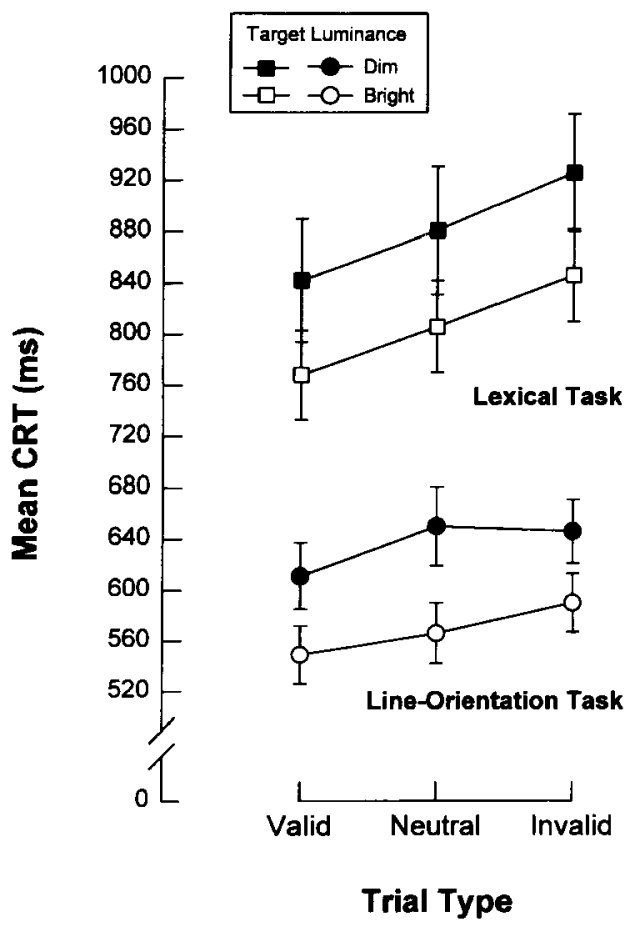

Figure 2. Mean CRTs on valid, neutral, and invalid trials in the lexical decision task (squares) and the line-orientation decision task (circles) with bright luminance targets (unfilled symbols) and dim luminance targets (filled symbols). Bars $= \pm 1 S E M$.
Table 2

Mean Accuracy Rate $(M)$ and Standard Error $(S E)$

(in Percent) as a Function of Age, Trial Type, Task Complexity, and Target Luminance

\begin{tabular}{|c|c|c|c|c|c|c|}
\hline \multirow{3}{*}{$\begin{array}{c}\text { Target } \\
\text { Luminance }\end{array}$} & \multicolumn{6}{|c|}{ Trial Type } \\
\hline & \multicolumn{2}{|c|}{ Valid } & \multicolumn{2}{|c|}{ Neutral } & \multicolumn{2}{|c|}{ Invalid } \\
\hline & $M$ & $S E$ & $M$ & $S E$ & $M$ & $S E$ \\
\hline \multicolumn{7}{|c|}{ Line-Orientation Task } \\
\hline \multicolumn{7}{|l|}{ Younger adults } \\
\hline Bright & 98.3 & 0.4 & 97.5 & 0.7 & 97.4 & 1.1 \\
\hline Dim & 97.4 & 0.4 & 97.3 & 1.2 & 98.0 & 0.6 \\
\hline \multicolumn{7}{|l|}{ Older adults } \\
\hline Bright & 99.4 & 0.2 & 98.6 & 0.6 & 98.8 & 0.4 \\
\hline Dim & 99.0 & 0.3 & 98.0 & 0.7 & 98.9 & 0.4 \\
\hline \multicolumn{7}{|c|}{ Lexical Task } \\
\hline \multicolumn{7}{|l|}{ Younger adults } \\
\hline Bright & 95.7 & 0.6 & 96.2 & 0.9 & 95.4 & 0.9 \\
\hline Dim & 95.0 & 0.6 & 95.3 & 0.8 & 94.8 & 0.9 \\
\hline \multicolumn{7}{|l|}{ Older adults } \\
\hline Bright & 98.2 & 0.4 & 98.4 & 0.5 & 98.0 & 0.5 \\
\hline Dim & 98.0 & 0.5 & 97.7 & 0.4 & 97.6 & 0.6 \\
\hline
\end{tabular}

the luminance effect on valid ( $63 \mathrm{msec})$, neutral $(83 \mathrm{msec})$, and invalid $(55 \mathrm{msec})$ trials revealed that the effect was significantly greater for neutral trials than for invalid trials; the other comparisons were not significant.

The significant trial type $\times$ task complexity interaction can be extrapolated from the data plotted in Figure 2. Bonferroni comparisons of the mean CRTs on valid, neutral, and invalid trials were conducted for each task separately. In the line-orientation task, mean CRTs on valid trials were significantly different from those on neutral and invalid trials, but mean CRTs on neutral and invalid trials were not significantly different. The mean differences were $28 \mathrm{msec}$ between valid and neutral trials, $38 \mathrm{msec}$ between valid and invalid trials, and $10 \mathrm{msec}$ between neutral and invalid trials. In the lexical task, all pairwise differences were significant. The mean differences were $38 \mathrm{msec}$ between valid and neutral trials, $81 \mathrm{msec}$ between valid and invalid trials, and $43 \mathrm{msec}$ between neutral and invalid trials.

\section{Accuracy}

A four-factor ANOVA was performed on the arcsintransformed accuracy rates with age as a between-subjects factor and trial type, task complexity, and target luminance as within-subjects factors. As before, luminance was nested within task complexity. The mean untransformed accuracy levels are provided in Table 2 . The analysis revealed significant main effects of age $[F(1,28)=$ $9.94, p<.01]$, trial type $[F(2,56)=3.92, p<.03]$, task complexity $[F(1,28)=39.92, p<.001]$, and luminance (task) $[F(2,56)=5.06, p<.01]$, and there were two significant two-way interactions [age $\times$ task complexity, $F(1,28)=8.58, p<.01$; trial type $\times$ task complexity, $F(2,56)=6.02, p<.01]$. No other interactions were significant $(p>.1)$.

The effect of luminance was examined for each task separately. For the line-orientation task, the difference in 
luminance did not significantly affect response accuracy $[F(1,28)=2.24, p>.14]$. The mean percentage of correct responses was 98.3 with bright targets and 98.1 with dim targets. For the lexical task, the luminance manipulation did have a significant effect on response accuracy $[F(1,28)=8.40, p<.01]$. The mean percentages of correct responses were 97.0 and 96.4 with bright and dim targets, respectively.

Follow-up tests of the significant trial type $\times$ task complexity interaction indicated that the simple effect of trial type was not significant in the lexical task $[F(2,56)=$ $1.87, p>.16]$, but it was significant in the line-orientation task $[F(2,56)=6.21, p<.01]$. The mean percentages of correct responses in the lexical task were $96.7,96.9$, and 96.4 on valid, neutral, and invalid trials, respectively. In the line-orientation task, these values were 98.6, 97.9, and 98.3 on valid, neutral, and invalid trials, respectively. A Tukey test of the differences between the trial type means in the line-orientation task indicated that response accuracy was significantly reduced for neutral trials relative to that for valid trials; no other differences were significant.

Regarding the significant age $\times$ task complexity interaction, follow-up tests revealed that older adults were significantly more accurate than were younger adults in both the line-orientation and the lexical tasks. In addition, both age groups responded more accurately in the line-orientation task than in the lexical task. The mean percentages of correct reponses for the older group were 98.8 in the line-orientation task and 98.0 in the lexical task, whereas for the younger group, these values were 97.7 and 95.4 , respectively. As these percentages suggest, the effect of task on response accuracy was found to be significantly greater for the younger adults than for the older adults.

\section{Additional Analyses}

To further examine the effects of age, task complexity, and target luminance on the allocation of processing resources during informative visuospatial precuing, a threefactor ANOVA was conducted using the difference in CRT performance on valid and invalid trials (i.e., invalidtrial CRT minus valid-trial CRT) as the dependent vari-

Table 3

Mean Magnitude $(M)$ and Standard Error $(S E)$ (in Milliseconds) of the Informative-Precuing Effect (Invalid-Trial CRTs - Valid-Trial CRTs) as a Function of Age, Task Complexity, and Target Luminance

\begin{tabular}{lllll}
\hline & \multicolumn{2}{c}{ Bright Targets } & \multicolumn{2}{c}{ Dim Targets } \\
\cline { 2 - 5 } Age & $M$ & $S E$ & $M$ & $S E$ \\
\hline \multirow{3}{*}{$\begin{array}{l}\text { Younger } \\
\text { Older }\end{array}$} & 42 & 11 & 36 & 7 \\
& 42 & 14 & 33 & 9 \\
Younger & 68 & 19 & 75 & 19 \\
Older & 88 & 15 & 91 & 18 \\
\hline
\end{tabular}

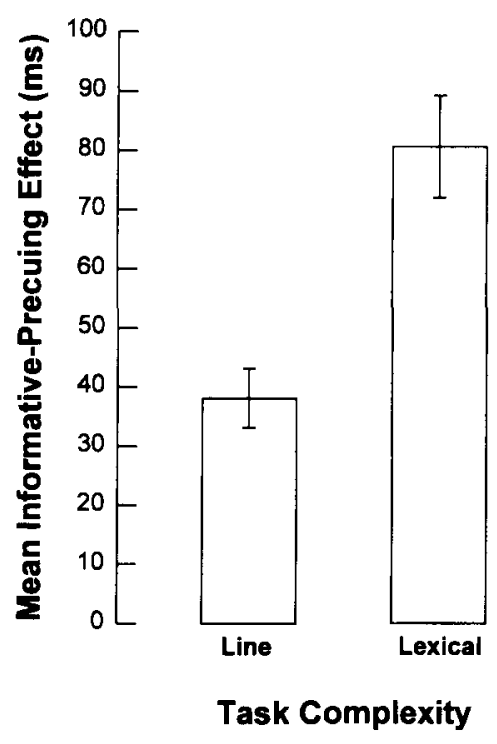

Figure 3. Mean informative-precuing effect (invalid-trial CRTs - valid-trial CRTs) for each level of task complexity. Bars $= \pm 1 S E M$.

able. Age was a between-subjects factor, and task complexity and target luminance were within-subjects factors, with luminance nested within task complexity. Table 3 lists the mean effect of informative precuing (the mean of the difference scores) for each condition. The analysis revealed a significant main effect of task complexity $[F(1,28)=17.41, p<.001]$. No other main effects or interactions were significant [age, $F(1,28)=$ 0.27 ; luminance (task), $F(2,56)=0.63$; age $\times$ task complexity, $F(1,28)=0.95$; age $\times$ luminance(task), $F(2,56)=$ 0.06]. As Figure 3 illustrates, the informative-precuing effect was considerably larger for the lexical task $(81 \mathrm{msec})$ than for the line-orientation task ( $38 \mathrm{msec})$.

Because the older adults' mean CRTs were, on average, longer than the younger adults' mean CRTs, a proportion score, as opposed to a difference score, may be more representative of the effects of age on the differential allocation of processing resources on informativeprecue trials. An additional three-factor ANOVA was performed that was identical to that described except using the proportion of change in CRT performance on invalid trials compared with valid trials [i.e., (invalidtrial CRT - valid-trial CRT)/valid-trial CRT] as the dependent variable. The outcome was the same as that with difference scores. The main effect of task was significant $[F(1,28)=5.12, p<.04]$. No other main effects or interactions were significant [age, $F(1,28)=0.64$; luminance (task), $F(2,56)=1.72$; age $\times$ task, $F(1,28)=0.15$; age $\times$ luminance (task), $F(2,56)=0.07]$.

It is possible that the absence of a significant age difference in the magnitude of the informative-precuing effect, using either difference or proportion scores, may be due to differential effort invested in the process of resource allocation over trial blocks. Older adults may have 
become fatigued over the course of the experiment, thus exerting less effort in allocating processing resources in response to the precue, relative to their younger counterparts. If, under conditions in which performance criteria are stressed, older adults compensate for reduced resource capacity by strategically allocating a greater share of available resources to the most probable (i.e., cued) target location on informative-precue trials and, thus, a lesser share to the less probable (i.e., uncued) target location, relative to younger adults, then the magnitude of the informative-precuing effect would be expected to be larger for the older group than for the younger group. Reduced effort in allocating resources to the cued location on the part of the older observers, brought about by fatigue, could have artificially deflated the magnitude of the informative-precuing effect, and this may have obscured significant age differences. Perhaps differences in allocation strategies over trial blocks were masked by collapsing over blocks within a condition.

To address this possibility, performance was compared on the first and last blocks of each condition (Blocks 1 and 4 for the line-orientation task, Blocks 1 and 6 for the lexical task). An ANOVA was performed using the difference scores (invalid-trial CRT - valid-trial CRT) as the dependent variable, with age as a between-subjects factor and task complexity and block (first, last) as within-subjects factors. Because in the previous analyses luminance was not found to influence the magnitude of the informative-precuing effect, it was not included as a factor here. This increased the number of invalid trials used in the computation of the difference score for each block. The results of the analysis mirrored those of the previous analyses: The only significant effect was a main effect of task complexity $[F(1,28)=11.98, p<.001]$. The critical age $\times$ block interaction failed to reach significance $[F(1,28)=2.28, p>.10]$. For the younger group, the means of the difference scores were $57 \mathrm{msec}(S E=$ $\pm 7 \mathrm{msec}$ ) for the first block and $76 \mathrm{msec}(S E= \pm 8 \mathrm{msec})$ for the last block. For the older group, the means were $56 \mathrm{msec}(S E= \pm 10 \mathrm{msec})$ and $57 \mathrm{msec}(S E= \pm 9 \mathrm{msec})$ for the first and last blocks, respectively.

Two additional three-factor ANOVAs were conducted to examine the possibility of differential allocation strategies due to fatigue. These analyses were identical to the one just reported except for the block factor. In one analysis, the block factor included average performance on practice blocks (which were at least half the number of trials as the experimental blocks) and average performance on experimental blocks. In the other analysis, the block factor included average performance on practice blocks and performance on the last experimental block. There were no differential effects due to age found in either analysis.

\section{DISCUSSION}

Several findings emerge from the latency data, and these are evident in Figure 4, which plots the mean CRTs

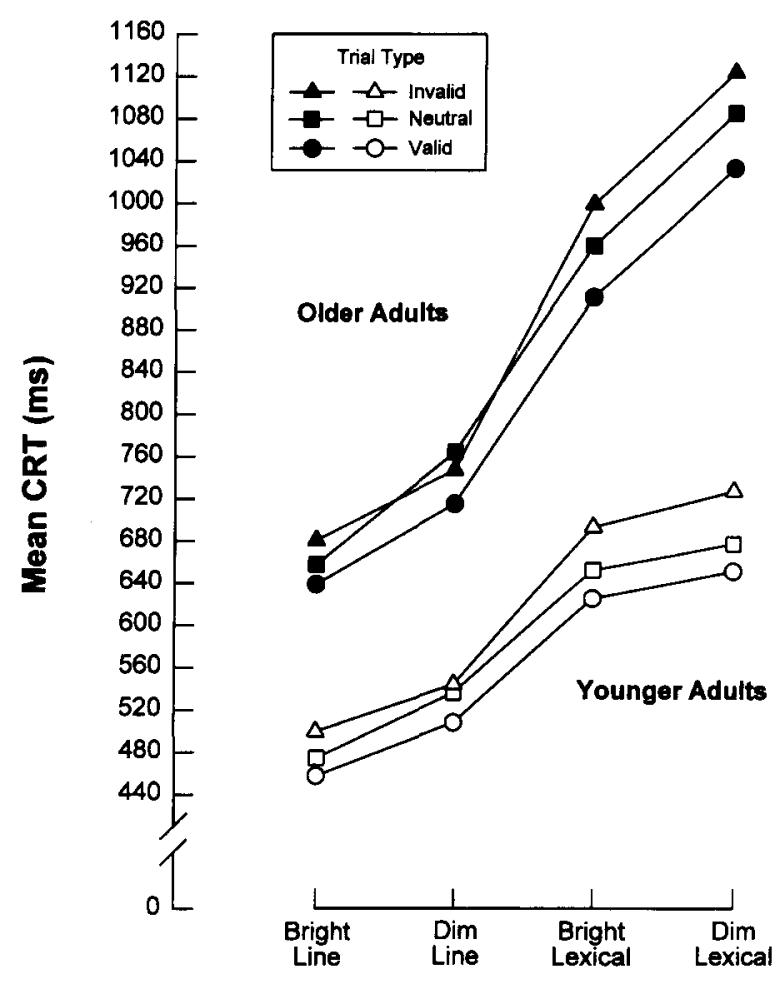

Figure 4. Mean CRTs of the younger (unfilled symbols) and older (filled symbols) groups on valid (circles), neutral (squares), and invalid (triangles) trials for the four levels of task demands (bright- and dim-luminance targets in the line-orientation and lexical decision tasks).

of the younger and older groups on valid, neutral, and invalid trials as a function of the variations in task demands produced by the levels of target luminance and task complexity. It was found that the response latency of the older group was consistently slower than that of the younger group, in line with the notion that the aging process produces a decrement in the speed of informationprocessing performance as measured by RT tasks (e.g., Wickens et al., 1987). As expected, increasing the task demands slowed response latency for both groups. In addition, the magnitude of response slowing was greater for the older group than for the younger group for both the decreases in target luminance and the increase in task complexity. This finding is consistent with the idea that the magnitude of age differences in performance speed increases with increases in the processing demands of the task (e.g., Salthouse, 1988, 1992b). The performance accuracy of the older group was consistently higher than that of the younger group (see Table 2), in line with other reports that older adults adopt more conservative response strategies (e.g., Bashore, Osman, \& Heffley, 1989). Although this finding suggests that the latency differences attributed to age may have been exaggerated, it is doubtful that differences in response accuracy are entirely responsible for the large CRT differences obtained. For example, the mean age difference in CRTs 
over all conditions was $273 \mathrm{msec}$, which is $46.5 \%$ of the younger group's mean CRT $(587 \mathrm{msec})$. The corresponding difference in response accuracy was $1.9 \%$ (42 out of 2,208 responses). ${ }^{4}$

The results involving age, target luminance, and task complexity reveal age sensitivity for both peripheral and central processes: The response latencies of the older group were more affected than were those of the younger group by decreasing target luminance-a peripheral, encoding-stage effect-and by increasing task complexity-a central, decision-stage effect. However, these results do not bear on the issue of differential age sensitivity for central and peripheral processes (see Kausler, 1991, for a brief review of this topic) because the task complexity manipulation was coupled with a luminance difference between the task stimuli (see Method section). In general, the luminance of the letter strings used in the lexical task was greater than that of the line segments used in the line-orientation task because the letter strings were larger than the line segments. Thus, the reported age difference in the effectiveness of the task-complexity manipulation may reflect peripheral, as well as central, stage effects. ${ }^{5}$

The precuing manipulation was successful in producing changes in CRTs under all levels of task demands for both age groups (see Figure 4). In the lexical task, the effects of luminance and precuing were additive. In the line-orientation task, decreasing luminance increased CRTs more on neutral trials than on invalid trials. This pattern is evident in the data of both groups, but it is more pronounced for the older group. For both bright and dim letter-string targets in the lexical task, CRTs were fastest on valid trials, slower on neutral trials, and slowest on invalid trials, and all the pairwise differences were significant. This outcome, which is consistent with other visuospatial precuing studies (e.g., Posner et al., 1980), was also found for the bright line-segment targets in the lineorientation task. However, for the dim line-segment targets, valid-trial CRTs were fastest, but neutral- and invalidtrial CRTs did not significantly differ. This resulted because of the greater slowing of CRTs on neutral trials, relative to that on invalid trials, with dim line-segment targets.

The magnitude of the informative-precuing effect was found to be dependent on the task: The effect was larger when the task involved lexical decisions than when it involved line-orientation decisions (see Figure 3 ). This finding can be accounted for by models that posit strategic variations in spatial resource allocation dependent on specific task parameters that influence task difficulty (e.g., Hawkins et al., 1988; LaBerge \& Brown, 1989). According to these accounts, if two tasks that differ in difficulty are presented in separate trial blocks, then resources may be distributed differently between tasks so that a larger effect size is associated with the more difficult task. In this experiment, the lexical task produced overall slower response rates and higher error rates than did the line-orientation task, indicating that it was the more difficult one. The tasks were presented in separate trial blocks, and the subjects were informed of the type of task at the beginning of each block. Given these considerations, the difference in precuing-effect size was likely due to a strategic change in the resource allocation policy. For example, a greater share of the available resource capacity may have been allocated to the cued location (and a lesser share allocated to the uncued location) in lexical task blocks than in line-orientation task blocks, thus enhancing the valid-invalid RT difference. But further research is needed to precisely specify the mechanism(s) underlying the between-task difference in precuing-effect size.

Significant interactions involving age and trial type were not found in the CRT data or the accuracy data, nor were there significant age effects found in the magnitude of the informative-precuing effect whether the dependent variable was the difference between valid-and invalidtrial CRTs or the proportion of change in CRT on invalid trials from valid-trial performance levels. There were no clear indications of age differences in allocation strategies attributable to fatigue, so this does not appear to account for the lack of age differences in precuing effects. It is possible that the lack of significant age differences may be due to differential responsivity to the informative precue between the older and younger groups. If, on the average, the older subjects were less responsive to the precue (i.e., were less likely to engage in the process of differentially allocating resources to the cued and uncued locations), then age differences may have been obscured. This is assuming that age-equivalent responsivity would result in a larger effect of precuing for the older group. The standard error data presented in Table 3 indicate that the variability in the size of the informativeprecuing effect was approximately equal for the two groups, which argues against the possibility of differential between-subject effect sizes (i.e., the possibility that some of the older subjects produced much smaller and others much larger precuing effects than did the younger subjects). Although this finding does not rule out the possibility of a general decrease in responsivity to the informative precue with age, it does suggest that the pattern of individual differences in responsivity does not dramatically change with age. The issue of betweengroup differences in responsivity to the critical manipulation is only partially addressed (and addressable) by the data in this study, in the aging literature in general, and in other studies that utilize a between-group design to make inferences about population differences. This important issue merits further study.

Although between-group differences in precuing were not statistically significant, the mean effect of informativeprecuing was larger for the older adults than for the younger adults in the lexical task conditions (see Table 3 ). The difference averaged over target luminance was $18 \mathrm{msec}$. This is a small effect size $(d=0.28)$, and a sample size of over 300 individuals per group would be needed to achieve a power of .8 to detect a population 
difference of this size as significant at $\alpha=.05$ (Cohen, 1992). A trend toward larger effects for older adults has also been reported in other studies involving the visuospatial attention system (e.g., Folk \& Hoyer, 1992; Hartley, 1993; Hartley et al., 1990; Nissen \& Corkin, 1985). This suggests that small sample sizes tested in those studies, and in this study, may be precluding a small but reliable age effect. However, this argument is weakened by other studies (e.g., Robin \& Rizzo, 1992) that reveal a trend in the opposite direction (i.e., smaller effects for the older group).

The aim of this experiment was to test the hypothesis that due to a reduction in processing resource capacity with age, precuing effects would differ between groups of younger and older adults, particularly under conditions of increased task demands in which the resource demand may exceed the resource capacity of the older but not the younger adults. The data do not provide strong support for this hypothesis. There were no observable age differences in precuing effects in the less demanding, line-orientation task conditions. In the more demanding, lexical task conditions the older group produced larger precuing effects than did the younger group, but the age-effect size was small and not statistically significant. These results are in contrast to the large age effects observed in CRT performance for the other manipulations. The mean differences in effect sizes between the younger and older groups were $31 \mathrm{msec}$ for luminance in the line-orientation task $(d=1.15)$, $96 \mathrm{msec}$ for luminance in the lexical task $(d=1.18)$, and $151 \mathrm{msec}$ for task complexity $(d=1.56)$. It is not clear whether age-related resource reduction can sufficiently account for the substantial age-related latency differences.

The most interesting finding in this study is that the pattern of CRT performance on valid, neutral, and invalid trials was similar for younger and older adults even though the performance pattern changed between the line-orientation and lexical tasks. ${ }^{6}$ This indicates that older and younger adults exhibit similar strategic variations in processing resource allocation across spatial locations, and it provides additional support for the idea that the processes involved in allocating resources to spatial locations remain relatively well preserved with advancing age (e.g., Folk \& Hoyer, 1992; Hartley, Kieley, \& McKenzie, 1992; Hartley et a!., 1990; Nissen \& Corkin, 1985; Robin \& Rizzo, 1992). This is consistent with Hartley's (1993) suggestion that the posterior brain attention system, as defined by Posner and Petersen (1990; also Posner \& Dehaene, 1994), may be unaffected by the aging process.

Overall, the outcome of this study is not consistent with the idea that a reduction in resource capacity (i.e., in the quantity of processing resources available for task performance) is the primary contributor to the agerelated slowing phenomenon (Salthouse, 1988, 1991, 1992a). Capacity reduction may occur with aging, but it appears to be minimal over those resource pools utilized for performance in the line-orientation and lexical decision tasks. From a resource perspective (e.g., Navon \&
Gopher, 1979, 1980), the data suggest that age-related slowing results primarily from differences in specific subject-task parameters (e.g., signal quality, manual dexterity) that produce changes in RT irrespective of any cognitive factors, coupled with a reduction in the processing efficiency of the resources invested in the task. Similar accounts have been proposed by other investigators (e.g., Myerson, Hale, Wagstaff, Poon, \& Smith, 1990).

\section{REFERENCES}

Allen, P. A., Madden, D. J., \& Crozier, L. C. (1991). Adult age differences in letter-level and word-level processing. Psychology \& Aging, 6, 261-271.

Allen, P. A., Madden, D. J., Weber, T. A., \& Groth, K. E. (1993). Influence of age and processing stage on visual word recognition. Psychology \& Aging, 8, 274-282.

Bashore, T. R., Osman, A., \& HefFley, E. F. (1989). Mental slowing in elderly persons: A cognitive psychophysiological analysis. Psychology \& Aging, 4, 235-244.

Bowles, N. L., \& Poon, L. W. (1981). The effect of age on speed of lexical access. Experimental Aging Research, 7, 417-426.

Carroll, J. B., Davies, P., \& Richman, B. (1971). The American heritage word frequency book. New York: American Heritage.

Cheal, M. L., \& Lyon, D. R. (1992). Benefits from attention depend on the target type in location-precued discrimination. Acta Psychologica, 81, 1-25.

CoHEN, J. (1992). A power primer. Psychological Bulletin, 112, $155-$ 159.

ERIKSEN, C. W., \& YEH, Y. (1985). Allocation of attention in the visual field. Journal of Experimental Psychology: Human Perception \& Performance, 11, 583-597.

FolK, C. L., \& HoYer, W. J. (1992). Aging and shifts of visual spatial attention. Psychology \& Aging, 7, 453-465.

HARTLEY, A. A. (1993). Evidence for the selective preservation of spatial selective attention in old age. Psychology \& Aging, 8, 371-379.

Hartley, A. A., Kieley, J., \& MCKenZie, C. R. M. (1992). Allocation of visual attention in younger and older adults. Perception \& Psychophysics, 52, 175-185.

Hartley, A. A., Kieley, J. M., \& Slabach, E. H. (1990). Age differences and similarities in the effects of cues and prompts. Journal of Experimental Psychology: Human Perception \& Performance, 16, 523-537.

Hawkins, H. L., Shafto, M. G., \& Richardson, K. (1988). Effects of target luminance and cue validity on the latency of visual detection. Perception \& Psychophysics, 44, 484-492.

Henderson, J. M. (1991). Stimulus discrimination following covert attentional orienting to an exogenous cue. Journal of Experimental Psychology: Human Perception \& Performance, 17, 91-106.

Hughes, H. C. (1984). Effects of flash luminance and positional expectancies on visual response latency. Perception \& Psychophysics, 36, 177-184

Johnson, D. N., \& YANTIS, S. (1995). Allocating visual attention: Tests of a two-process model. Journal of Experimental Psychology: Human Perception \& Performance, 21, 1376-1390.

Kahneman, D. (1973). Attention and effort. Englewood Cliffs, NJ: Prentice-Hall.

Kaswan, J., \& Young, S. (1965). Effect of luminance, exposure duration and task complexity on reaction time. Journal of Experimental Psychology, 69, 393-394.

KAUSLER, D. H. (1991). Experimental psychology, cognition and human aging (2nd ed.). New York: Springer-Verlag.

KIRK, R. E. (1982). Experimental design (2nd ed.). Monterey, CA: Brooks/Cole.

Kline, D. W., \& Schieber, F. (1985). Vision and aging. In J. E. Birren \& K. W. Schaie (Eds.), Handbook of the psychology of aging (2nd ed., pp. 296-331). New York: Van Nostrand Reinhold.

LABERGE, D., \& BRown, V. (1989). Theory of attentional operations in shape identification. Psychological Review, 96, 101-124.

Lima, S., Hale, S., \& Myerson, J. (1991). How general is general 
slowing? Evidence from the lexical domain. Psychology \& Aging, 6, 416-425.

MADDEN, D. J. (1992). Selective attention and visual search: Revision of an allocation model and application to age differences. Journal of Experimental Psychology: Human Perception \& Performance, $18,821-836$

Maylor, E. A., \& RabBitt, P. M. A. (1994). Applying Brinley plots to individuals: Effects of aging on performance distributions in two speeded tasks. Psychology \& Aging, 9, 224-230.

MoraY, N. (1967). Where is capacity limited? A survey and a model. Acta Psychologica, 27, 84-92.

Myerson, J., Hale, S., Wagstaff, D., Poon, L. W., \& Smith, D. A. (1990). The information-loss model: A mathematical theory of agerelated cognitive slowing. Psychological Review, 97, 475-487.

Navon, D., \& Gopher, D. (1979). On the economy of the humanprocessing system. Psychological Review, 86, 214-255.

NAvon, D., \& Gopher, D. (1980). Task difficulty, resources, and dual task performance. In R. S. Nickerson (Ed.), Attention and performance VIII (pp. 297-315). Hillsdale, NJ: Erlbaum.

Nissen, M. J., \& Corkin, S. (1985). Effectiveness of attentional cueing in older and younger adults. Journal of Gerontology, 40, 185191

Posner, M. I., \& Dehaene, S. (1994). Attentional networks. Trends in Neurosciences, 17, 75-79.

Posner, M. I., Inhoff, A. W., Friedrich, F. J., \& Cohen, A. (1987). Isolating attentional systems: A cognitive-anatomical analysis. Psychobiology, 15, 107-121.

Posner, M. I., \& Petersen, S. E. (1990). The attention system of the human brain. Annual Review of Neuroscience, 13, 25-42.

Posner, M. I., SNyder, C. R. R., \& Davidson, B. J. (1980). Attention and the detection of signals. Journal of Experimental Psychology: General, 109, 160-174.

RATCLIFF, R. (1979). Group reaction time distributions and an analysis of distribution statistics. Psychological Bulletin, 86, 446-461.

RobiN, D. A., \& Rizzo, M. (1992). Orienting attention in audition and between audition and vision: Young and elderly subjects. Journal of Speech \& Hearing Research, 35, 701-707.

RoBINSON, D. L.. \& KERTZMAN, C. (1990). Visuospatial attention: Effects of age, gender, and spatial reference. Neuropsychologia, 28, 391-401.

SAlthouse, T. A. (1988). Resource-reduction intepretations of cognitive aging. Developmental Review, 8, 238-272.

SALTHOUSE, T. A. (1991). Theoretical perspectives on cognitive aging. Hillsdale, NJ: Erlbaum.

Salthouse, T. A. (1992a). The information-processing perspective on cognitive aging. In R. J. Sternberg \& C. A. Berg (Eds.), Intellectual development (pp. 261-277). New York: Cambridge University Press.

SALTHOUSE, T. A. (1992b). Why do adult age differences increase with task complexity? Developmental Psychology, 28, 905-918

SHAW, M. L. (1978). A capacity allocation model for reaction time. Journal of Experimental Psychology: Human Perception \& Performance, 4, 586-598.

Shaw, M. L., \& Shaw, P. (1977). Optimal allocation of cognitive resources to spatial locations. Journal of Experimental Psychology: Human Perception \& Performance, 3, 201-211.

Snodgrass, J. G., Levy-Berger, G., \& Haydon, M. (1985). Human experimental psychology. New York: Oxford University Press.

Somberg, B. J., \& Salthouse, T. A. (1982). Divided attention abilities in young and old adults. Journal of Experimental Psychology: Human Perception \& Performance, 8, 651-663.

StURR, J. F., van Orden, K., \& TaUb, H. A. (1987). Selective attenuation in brightness for brief stimuli and at low intensities supports age-related transient channel losses. Experimental Aging Research, 13, 145-149

WeAle, R. A. (1963). The aging eye. New York: Harper

WEALE, R. A. (1982). Senile ocular changes, cell death and vision. In R. Sekuler, D. Kline, \& K. Dismukes (Eds.), Aging and human visual function (pp. 161-171). New York: Alan R. Liss.

WiCkENS, C. D. (1980). The structure of attentional resources. In R. S.
Nickerson (Ed.), Attention and performance VIII (pp. 239-257). Hillsdale, NJ: Erlbaum.

Wickens, C. D. (1984). Processing resources in attention. In R. Parasuraman \& R. D. Davies (Eds.), Varieties of attention (pp. 63-102). Orlando, FL: Academic Press.

WICKENS, C. D. (1987). Attention. In P. A. Hancock (Ed.), Human factors psychology (pp. 29-80). Amsterdam: Elsevier.

Wickens, C. D., BRaune, R., \& Stokes, A. (1987). Age differences in the speed and capacity of information processing: 1 . A dual-task approach. Psychology \& Aging, 2, 70-78.

\section{NOTES}

1. The terms processing resources and attention are often used interchangeably. However, in this report, we use processing resources to refer exclusively to those units essential for performing the computations underlying information processing, and we use attention to refer to a system of cognitive control. The attention system appears to be composed of several functionally interrelated, anatomically distinct subsystems. One of these-the posterior attention system-functions in the allocation of processing resources to information originating from particular spatial locations (e.g., Posner \& Dehaene, 1994; Posner \& Petersen, 1990).

2. There were about twice as many trials of the lexical task $(1,440)$ as of the line-orientation task (768). This is because we initially planned to analyze performance on word and pseudoword trials as separate levels of task complexity. The rationale for this stemmed from reports (e.g., LaBerge \& Brown, 1989) indicating that words are processed more automatically than are pseudowords, and, thus, fewer processing steps may be required to identify a letter string as a word than as a pseudoword. On the advice of a reviewer, we simplified the design by averaging across word and pseudoword trials for the analyses.

3. The geometric means, rather than the arithmetic means, of the CRTs were used in the analyses. The geometric mean is the antilogarithm of the arithmetic mean of the logarithms of the RTs. The arithmetic mean is the proper measure of central tendency for a normal distribution of scores, but RTs are not normally distributed. The distribution is almost always skewed to the right with occasional very long outlier RTs (Ratcliff, 1979). When distributions are skewed to the right, the median or the geometric mean tend to be the preferred measures of central tendency (Snodgrass, Levy-Berger, \& Haydon, 1985 ). A problem with the median, however, is that it has rather low precision relative to the geometric mean. An alternative approach to dealing with skewed distributions having outliers is to throw out "unreasonably long" RTs (trimming) or to replace them with the next largest value (Winsorizing) (Snodgrass et al., 1985). Because a uniform definition of "unreasonably long" does not currently exist, we rejected trimming or Winsorizing as too subjective. The values presented in the tables and figures are the arithmetic means of the geometric mean CRTs.

4. The possibility of a between-group speed-accuracy tradeoff was not checked empirically because this would have required a comparison of the groups' speed-accuracy tradeoff functions, which we had not obtained. Without these functions, the relationship between speed and accuracy across the groups cannot be precisely evaluated.

5. There are two additional considerations that complicate attempts at specifying the effects of age on central versus peripheral processing stages, especially when using manipulations such as those used in this study (i.e, luminance and task complexity). First, because of the many changes in the eye with aging, older and younger adults may be differentially sensitive to suprathreshold stimulus intensities (Weale, 1963, 1982). Sturr, van Orden, and Taub (1987), for example, found age differences in the functions relating stimulus intensity and perceived brightness. So, even when stimuli are equated for physical intensity, age differences in perceived intensity may exist, and these differences may produce differences in mean response latencies irrespective of any other stimulus or task manipulations. Second, Kaswan and Young (1965) showed that differences in task complexity produce changes in the shape of the function relating CRT and stimulus luminance. Thus, manipulations of luminance and task complexity may 
have interactive effects on CRT. This, coupled with the possible age differences in CRT due to differences in perceived stimulus luminance, makes it difficult to parcel and compare age effects at central and peripheral processing stages.

6 . We examined this statistically by comparing the difference in CRTs between tasks on valid, neutral, and invalid trials. A two-way ANOVA was performed on the difference scores (e.g., valid-trial CRTs in the lexical task minus valid-trial CRTs in the line-orientation task, etc.) with age as a between-subjects factor and trial type as a within-subjects factor. The main effects of age $[F(1,28)=18.33]$ and trial type $[F(2,56)=13.15]$ were significant $(p s<.001)$, but the age $\times$ trial type interaction was not $[F(2,56)=0.66, p>.51]$. A Tukey test indicated that the CRT difference between tasks on invalid trials $(268 \mathrm{msec})$ was significantly different from both the neutral-trial difference $(235 \mathrm{msec})$ and the valid-trial difference $(225 \mathrm{msec})$; the valid- and neutral-trial differences did not differ.

(Manuscript received June 29, 1993;

revision accepted for publication October 22, 1995.) 